\title{
First come, first served? Influence of changed object configuration on object-based attention
}

\author{
San-Yuan Lin $\cdot$ Su-Ling Yeh
}

Published online: 30 December 2010

(C) Psychonomic Society, Inc. 2010

\begin{abstract}
Evidence for object-based attention is based mainly on studies using object displays that remain unchanged throughout, with the assumption that object representation should be completed and stabilized before it is selected for further processing. We used the modified double-rectangle cuing paradigm of Egly, Driver, and Rafal (Journal of Experimental Psychology: General, 123, 161-177, 1994) but introduced a configuration change to the cued-object display to test whether object-based attention is determined by the cued- or, alternatively, the changed-object display. Four small rectangles were presented in the initial display; critically, after one was cued, an occluder was presented to make the four small rectangles amodally completed into the double-rectangle configuration. Results show that objectbased attention is determined by the changed display, but not by the cued display. This suggests that object-based attention is an interactively evolving process between object representation and attention, rather than a serial process in which attention operates after object representation is completed.
\end{abstract}

Keywords Object-based attention · Visual perception . Precuing

Selective processing is an important function of attention that prevents the brain from being overwhelmed by vast amounts of information. Previous studies have shown that selection can be based on objects (Duncan, 1984; Vecera \& Farah, 1994), in addition to spatial locations (Eriksen \& Yeh, 1985; Posner, Snyder, \& Davidson, 1980), as demonstrated

\footnotetext{
S.-Y. Lin · S.-L. Yeh $(\bowtie)$

Department of Psychology / Center for Neurobiology

and Cognitive Science, National Taiwan University,

No. 1, Sec. 4, Roosevelt Rd.,

Taipei, Taiwan 10617

e-mail: suling@ntu.edu.tw
}

in the popular double-rectangle cuing paradigm of Egly, Driver, and Rafal (1994). In this paradigm, two parallel rectangles are presented, and one end of them is brightened as a cue to indicate the possible location of the target - a small solid square - which is shown subsequently within one end of a rectangle. Existence of object-based attention is indicated by faster detection when the target appears at an uncued end that belongs to the same object as the cued end than when it appears at the uncued end that belongs to the other object, with identical cue-to-target distance between the two conditions (i.e., the same-object effect).

When attention is directed to the cued end in this paradigm, it is reasonable to infer that object-based attention relies on completed or stabilized object representation and that it should be this cued object that is selected for further processing (i.e., the cued-object hypothesis). Several lines of study are consistent with this hypothesis. For example, it has been shown that a sufficiently long preview time for the initial display-presumably used for stabilizing the object representation of the to-be-cued object is necessary for object-based attention (Shomstein \& Behrmann, 2008). Furthermore, a cued object, as compared with an uncued one, is more likely to influence the distribution of attention (Alvarez \& Scholl, 2005; Kravitz \& Behrmann, 2008) and enjoys more attentional resource as the cue-totarget interval increases (Benso, Turatto, Mascetti, \& Umiltà, 1998; Shomstein \& Yantis, 2004). However, it remains unknown whether object-based attention would be affected if a change were made after attention had been cued to a particular object (i.e., the cued-object hypothesis is violated); studies using the double-rectangle paradigm to examine object-based attention mainly use unchanged object displays upon which attention is directed (e.g., Abrams \& Law, 2002; Lamy \& Tsal, 2000; Macquistan, 1997; Shomstein \& Yantis, 2004; for a review, see Scholl, 2001). This may be due to 
certain implicit assumptions regarding how attention interacts with object representation; for example, any change occurring to the object after it has been cued might erase the attentional processing that is accruing on the configuration of the cued object. Alternatively, attentional processing might be unfolding unidirectionally, so that any changes induced after cuing are simply unable to affect the already-initiated attentional processing.

Facing a constantly changing visual world, however, retinal inputs often change, and sometimes such changes result in the change of object boundaries (e.g., an object split into two, or two objects merge into one). These changes should be incorporated into the existing representation and should interact with attentional processing (i.e., the dynamic-updating hypothesis). If our visual system were to sample only initial snapshots of the selected object, by the time an action was executed upon the object, the information about the initially selected - but later changedobject would be inaccurate, resulting in a failure of visually guided action. Indeed, it has been shown that when facing a dynamic change in the visual field, people can keep track of changes of object positions or features (Kahnemann, Treisman, \& Gibbs, 1992; Kellie \& Shapiro, 2004; Moore, Mordokoff, \& Enns, 2007; Pylyshyn \& Storm, 1988; Raymond, 2003). For example, when presented with several identical discs that move within a display, participants in Pylyshyn and Storm could track position changes of up to four discs when these targets were briefly flashed before all eight discs started to change positions.

In the present study, we pit the dynamic-updating hypothesis against the cued-object hypothesis by introducing a change to the cued object. Specifically, the dynamicupdating hypothesis proposes that, instead of relying on a completed or stabilized object representation, object-based attention is an iterative process consisting of feedback loops that continuously integrate the changes in the objects into the initially attended object representation and lasts until an action (e.g., a keypress) is carried out. When a change occurs to a cued object, the change triggers attention to reenter the object representation that is being updated and interacts with this changed information. Such an interaction could be achieved by a reentrant process built into the interconnectivity between levels of visual areas (Di Lollo, Enns, \& Rensink, 2000). In this regard, the dynamicupdating hypothesis can be viewed as a conceptual extension of the cued-object hypothesis. What we emphasize here is that the object representation upon which selection is based is not static but, rather, dynamic.

Furthermore, as was suggested by Goldsmith and Yeari (2003), object-based attention is affected by whether attentional focus covers the objects. Using the doublerectangle paradigm, their participants were instructed to maintain their focus wide open to cover the two rectangles or to narrowly focus on the central fixation until a response was made. Only when attentional focus was enlarged to cover the two rectangles was object-based attention found. They suggested that the dynamics in a trial, such as the onset of the cue and the change in the object, may alter the attentional focus on the objects, which, in turn, would influence object-based attention. It is thus possible that the initial representation of the cued object is constantly monitored by the reentrant process before a response is executed. Once a response-for example, a keypress for target detection - is programmed, the status of the object representation is accessed and updated by the reentrant processing. Hence, changes made before a response is carried out will have a chance to be integrated into the existing object representation, thereby influencing the operation of object-based attention.

To test the dynamic-updating hypothesis, we adopted a version of the double-rectangle paradigm as modified by Pratt and Sekuler (2001). In their display, part of an object was occluded from view by another object, but the occluded object was perceived as being continuous behind it (amodal completion): The initial display of four small rectangles was perceived as two larger rectangles after an occluder had been added. Pratt and Sekuler introduced amodal completion before the cue; however, we delayed it until after one object was cued. In this arrangement, we had a cued display with four objects that differed from the changed display, which had two amodally completed objects. The cue-target relationship that defined the sameobject effect was arranged with regard to the changed display; according to the dynamic-updating hypothesis, the same-object effect should be observed.

\section{Experiment 1}

Although it has been suggested that visual completion occurs preattentively (Mattingley, Davis, \& Driver, 1997; Rensink \& Enns, 1998), it has also been suggested that amodal completion takes time-about $100 \mathrm{~ms}$ - to accomplish (Sekuler \& Palmer, 1992). To ensure that the strength of the changed object representation was sufficient to interact with attention when the target appeared, in this experiment we introduced an occluder via a pictorial depth cue $100 \mathrm{~ms}$ before the target display. Also, according to the finding of Goldsmith and Yeari (2003) that object-based attention is obtained only when the attentional focus is wide open to cover the objects, we added a peripheral flash that consisted of four L-shaped patterns before the cue display to ensure that participant's attentional focus was expanded to cover the four small rectangles in the cue display (see also Ho \& Yeh, 2009). Therefore, any object effects obtained in favor of the 
dynamic-updating hypothesis should not be attributed to ignorance of the cued object.

\section{Method}

Participants A group of 23 students from National Taiwan University was recruited in return for a receipt of course credit. All had normal or corrected-to-normal vision and were naïve as to the purpose of the experiment.

Apparatus The stimuli were displayed on a monitor (Eizo F552-M) with $640 \times 480$ resolution and capable of producing 256 levels of grayscale. The DMDX program (Forster \& Forster, 2003) was used to present the stimuli and collect the timed responses. The participants sat in a dimly lit chamber at a viewing distance of $57 \mathrm{~cm}$, with head position maintained by a chinrest.

Stimuli and procedure As is shown in Fig. 1, the initial display consisted of four small rectangles; each subtended $3.15^{\circ} \times 1.4^{\circ}$ (thickness, $0.2^{\circ}$; light gray), with a central plus pattern $\left(0.25^{\circ} \times 0.25^{\circ}\right.$; thickness, $0.04^{\circ}$; light gray $)$ as fixation.
The end-to-end distances of these rectangles were the same (8.8 $8^{\circ}$, except diagonal). The peripheral flash consisted of four L-shaped patterns $\left(2.4^{\circ} \times 2.4^{\circ}\right.$; thickness, $0.55^{\circ}$; black $)$. The cue was a U-shaped pattern $\left(1.4^{\circ} \times 1.4^{\circ}\right.$; thickness, $0.2^{\circ}$; white) that brightened three sides of a rectangle. An occluder $\left(11^{\circ} \times 3^{\circ}\right.$; dark gray) was shown to cover the inner four edges of the rectangles (no disparity-induced stereo). The target was a filled square $\left(1.4^{\circ} \times 1.4^{\circ}\right.$; light gray $)$ shown at one end of the amodally completed rectangles.

On each trial, the initial display appeared for 1,000 ms; then the peripheral flash appeared briefly $(33 \mathrm{~ms})$, and the previous display was restored $(66 \mathrm{~ms})$. Afterward, a cue appeared on one of the four outer ends $(100 \mathrm{~ms})$, and the initial display was restored $(100 \mathrm{~ms})$, followed by an occluder covering the four inner edges of the rectangles. The target then appeared on one end. The participant performed a timed detection task by quickly pressing the space bar, and the target-to-response reaction time (RT) was recorded. The target display disappeared after the response. On the target-absent (catch) trials, no response was required, and the display disappeared after a 1,000-ms time-out. The next trial started automatically.
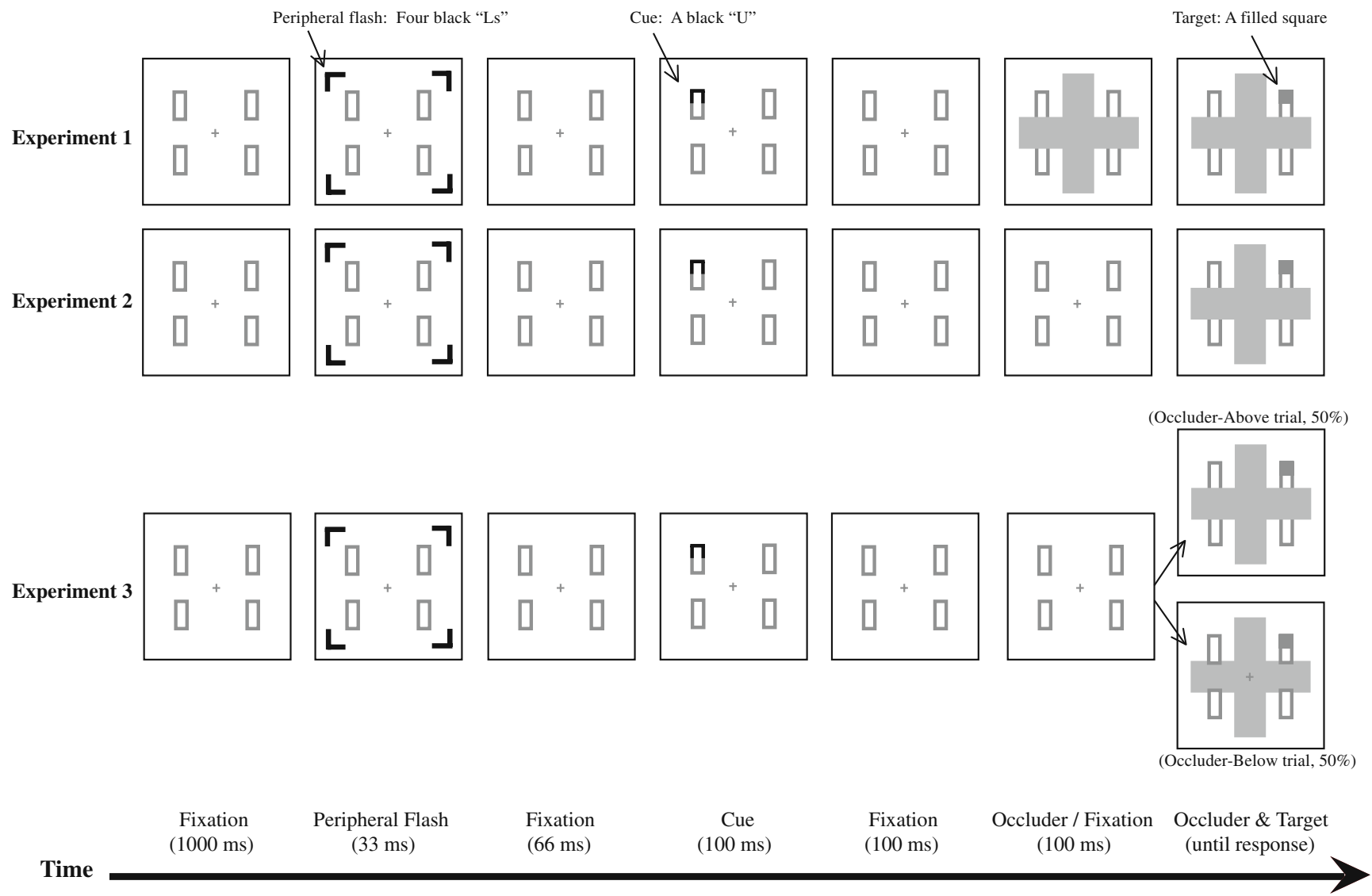

Fig. 1 Stimuli and experimental procedures. The occluder appeared after the cue, covering the four inner edges of the four small rectangles (i.e., amodal completion) in Experiments 1 and 2. The occluder appeared either above or below the rectangles in Experiment 3 (not shown to scale here, and the polarity of the display is inverted) 


\section{Design}

The cue appeared at one of the four outer ends of the rectangles with equal probability. On valid trials (240 trials, $75 \%$ cue validity), the target appeared at the cued location. On invalid-same trials (40 trials), the target appeared on the rectangle that formed an amodally completed rectangle with the cued rectangle. On invalid-different trials (40 trials), the target appeared on the rectangle that formed the other amodally completed rectangle. The target never appeared on the end that was diagonal to the cue. Half the trials were vertical rectangles (configuration after amodal completion), and the other half featured horizontal rectangles. To discourage preparatory responses, there were 64 catch trials (no target). The order of the trials was randomized. A practice session (20 trials) was administered to familiarize the participants with the procedure, and the data from this session were excluded from analysis.

Results and discussion

All anticipatory responses (RT $<150 \mathrm{~ms}$ ), misses (RT $>$ $1,000 \mathrm{~ms}$ ), and false alarms (responses to catch trials) were considered errors and were excluded from analysis. Fewer than $3 \%$ of the total trials were removed. Table 1 lists the mean RTs under each condition. We analyzed the location effect and the object effect separately (see also Egly et al., 1994; Shomstein \& Yantis, 2004). The location effect was examined by a one-way analysis of variance (ANOVA), with validity (valid vs. invalid) as the within-subjects factor. The result showed a significant main effect of validity, $F(1,22)=61.7, M S E=581.6, p<.001:$ RTs were shorter for valid trials than for invalid trials. The same-object effect was examined by conducting an ANOVA on the invalid trials (invalid-same vs. invalid-different). The result showed a significant main effect, $F(1,22)=14.7, M S E=202.3$, $p<.001$, Cohen's $f=0.54$ : RTs were shorter for invalid-same trials than for invalid-different trials. The presence of the same-object effect supports the dynamic-updating hypothesis.

However, one might suspect that the manipulation of presenting the occluder $100 \mathrm{~ms}$ before the target resulted in a trade-off in the strength of the object representations between the cued- and changed-object configurations: The time allowing for attention to interact with the cued object was $200 \mathrm{~ms}$, whereas that with the changed object, on average, was more than $400 \mathrm{~ms}$ (including the RT to the target). Thus, the main cause for the finding of object-based attention in favor of the dynamic-updating hypothesis in this experiment might have been the insufficient time for object representation of the cued object and for attention to engage and process the cued object. We examined this possibility in the next experiment.

\section{Experiment 2}

In this experiment, the change induced by amodal completion was delayed until the final frame in which the target was shown. This allowed more time for the formation of the cued-object representation upon which selection and processing is based, according to the cued-object hypothesis. By delaying amodal completion to the target frame, the cued-object configuration was presented for most of the time in a trial. As with Experiment 1, the dynamic-updating hypothesis predicts the same-object effect to be observed in this experiment.

\section{Method}

Participants Another group of 32 students from National Taiwan University was recruited and received course credit for participation. All had normal or corrected-tonormal vision and were naïve as to the purpose of the experiment.

Stimuli and procedure The stimuli and procedure were the same as those in Experiment 1, except that the presentation of the occluder was delayed until the final frame.

\section{Results}

The analysis was the same as that in Experiment 1. Error trials (fewer than 1.5\%) were excluded from further analysis. The location effect (valid vs. invalid trials) was significant, $F(1,31)=241.5, M S E=435.2, p<.0001$, and so was the same-object effect (invalid-same vs. invaliddifferent trials), $F(1,31)=10.9, M S E=246.7, p<.005$, Cohen's $f=0.39$.

Table 1 The mean reaction times (in milliseconds) and standard errors (in parentheses)

\begin{tabular}{|c|c|c|c|c|c|c|}
\hline & & Valid & Invalid-Same & Invalid-Different & Space Effect & Object Effect \\
\hline Experiment 1 & & $371.6(16.4)$ & $419.4(17)$ & $435.5(18.4)$ & 55.9 & 16.1 \\
\hline Experiment 2 & & $390.6(6.3)$ & $465.1(7.0)$ & $478.1(8.9)$ & 81.0 & 13.0 \\
\hline \multirow[t]{2}{*}{ Experiment 3} & Occluder-Above & $377.7(6.3)$ & $439.6(6.8)$ & $451.2(7.6)$ & 67.7 & 11.6 \\
\hline & Occluder-Below & $375(6.3)$ & $437.6(7.9)$ & $440.9(8.5)$ & 64.2 & 3.3 \\
\hline
\end{tabular}




\section{Discussion}

We still obtained the same-object effect when the presentation time of the initial four-rectangle display was lengthened to make the cued object visible for $300 \mathrm{~ms}$. Thus, it is unlikely that insufficient time given for attention to engage and process an object representation based on the cued object is reason to dispute the cued-object hypothesis.

However, there is another possible confounding factor that needs to be ruled out before a conclusion can be drawn. In Experiments 1 and 2, the occluder was always shown on top of the four rectangles, which induced the doublerectangle configuration through amodal completion. Over many trials, it may have induced an expectation that the double-rectangle configuration would emerge on every trial; thus, the participants may have simply ignored the relationship between the occluder and the four rectangles and may have treated the rectangles as if already grouped. If this were the case, the same-object effect we obtained might have been caused by such an expectation, rather than being attributable to attention's being allocated to the changed object. We tested this in the next experiment.

\section{Experiment 3}

To rule out the possibility that an expectation that developed through repeated trials influenced how participants organized the display and, thus, affected objectbased attention (Behrmann, Zemel, \& Mozer, 1998), in Experiment 3, the occluder either occluded the four rectangles or appeared behind them with equal probability, so that amodal completion occurred only on half the trials. We expected the same-object effect to be obtained only when the occluder appeared above - but not beneath - the four rectangles.

\section{Method}

Participants Another group of 60 students was recruited from National Taiwan University in return for a receipt of course credit. All had normal or corrected-to-normal vision and were naïve as to the purpose of the experiment.

Stimuli, procedure, and design The stimuli, procedure, and design were the same as those in Experiment 2, with the following changes: The occluder was enlarged-from $11^{\circ} \times$ $3^{\circ}$ to $11^{\circ} \times 4^{\circ}$ - to cover more than just the inner ends, as it did in Experiments 1 and 2. The occluder appeared to occlude the four rectangles (occluder-above condition) or to appear beneath them (occluder-below condition) with equal probability. The trials were divided into two blocks of equal trial distribution, separated by a rest. The order of the trials was randomized within each block. Because the occluderabove and occluder-below trials were mixed, the first block was used to familiarize the participants with the procedure and was not included in the analysis.

\section{Results and discussion}

Error trials were excluded from further analysis (fewer than $0.84 \%$ and $0.88 \%$ of total trials in the occluder-above and occluder-below conditions, respectively). A two-way ANOVA was performed on RT, with the within-subjects factors of cue validity (valid, invalid) and occluder position (occluder-above, occluder-below). Main effects of cue validity, $F(1,59)=380.8, M S E=686.3, p<.001$, and occluder position, $F(1,59)=6.823, M S E=172.5, p<.02$, were found, but there was no interaction, $F(1,59)=0.871$, $M S E=211, p=.35$. That is, the location effect was found regardless of occluder position: The mean RT of valid trials was shorter than that of invalid trials in both the occluderabove and occluder-below conditions.

The same-object effect was also examined by a two-way ANOVA with trial type (invalid-same, invalid-different) and occluder position (occluder-above, occluder-below) as within-subjects factors. The main effect of trial type, $F(1,59)=7.77, M S E=428.9, p<.01$, Cohen's $f=0.17$, was significant, and that of occluder position, $F(1,59)=3.56$, $M S E=642.9, p=.063$, was marginal. There was an interaction of trial type and occluder position, $F(1,59)=$ 4.05, MSE $=253.96, p<.05$. Planned $t$ tests showed that RTs for invalid-same trials were shorter than those for invaliddifferent trials (i.e., the object effect) only in the occluderabove condition, $t(59)=3.91, p<.001$, Cohen's $d=0.21$, but not in the occluder-below condition, $t(59)=0.88, p=.37$.

Thus, it is clear that the same-object effect was obtained only when the configuration made amodal completion possible. We excluded the possibility that an expectation might be accumulated over many trials about the outcome of the change in the target display by mixing the occluderabove and occluder-below trials, and yet the same-object effect was still found.

A close inspection of the RT pattern among invalid-same and invalid-different trials in this experiment reveals that the mean RT for invalid-same trials in the occluder-above conditions was not the shortest; on the contrary, the invaliddifferent trials in the occluder-above condition were the slowest (Table 1). Note that in a similar arrangement, Pratt and Sekuler (2001, Experiment 3) obtained the same RT pattern as we did in this experiment. Such an RT pattern, however, seems to counter the generally accepted idea that object-based attention is supported mainly by a same-object benefit: better processing for locations on the cued object than on the uncued object. We suspect that the reason for this RT pattern is that the presentation of the occluder at the 
target frame elevates overall RTs in the occluder-above condition, as compared with those in the occluder-below condition, where no amodal completion is expected to form. It is possible that amodal completion occurs in a partially parallel way, so that, in the occluder-above condition, the expected faster response at the invalid-same location was slowed down by such a partially parallel amodal completion. (For a detailed discussion of the amodal completion process in Experiments 2 and 3, please refer to the "Amodal Completion and Attentional Processing" section in the General Discussion.) Similarly, the unexpected slower response at the invalid-different location was also caused by this amodal completion process, with the amount of time being equal to the increase in RT for the invalidsame trials.

\section{General discussion}

In the present study, we introduced a change (amodal completion) after an object was cued in order to test the dynamic-updating hypothesis and the cued-object hypothesis. In three experiments, we demonstrated that objectbased attention was influenced by an object configuration that was changed after attention had been cued to it: when $200 \mathrm{~ms}$ (Experiment 1) or $300 \mathrm{~ms}$ (Experiment 2) was given for attention to interact with the cued display, and when the expectation about the outcome of change was ruled out (Experiment 3).

The results support the dynamic-updating hypothesis over the cued-object hypothesis. It suggests that when attention selects an object for further processing, attention and object representation interact until a response is made. This extends the idea of reentrant processing in object representation of Di Lollo et al. (2000); that is, attention operates on a representation that is constantly being updated, and changes made to the initial object display can also be incorporated into the existing object representation and benefit from attentional processing. This fits with our daily experience that many objects in the visual world change shape (e.g., being occluded, having reappeared, being split, or being merged) after we attend to them. Object-based attention, as has been shown in this study, can tolerate and further incorporate such changes.

Amodal completion and attentional processing

One may ask how, if it takes 75-100 ms for amodal completion to occur (e.g., Murray, Sekuler, \& Bennett, 2001; Sekuler \& Pratt, 1992), we could have obtained the same-object effect in Experiments 2 and 3, where the occluder was introduced at the final frame with the target, leaving seemingly no time for amodal completion to occur?
It is possible that amodal completion is processed in parallel with other ongoing processes (e.g., the act of summoning attention to reenter the updated representation and the initiation of motor programming for the keypress response); thus, the time needed for amodal completion normally observed in the aforementioned studies overlaps with these ongoing processes. Compare the RTs in Experiment 1 (where the occluder was presented $100 \mathrm{~ms}$ before the target) with those in Experiments 2 and 3 (where the occluder was presented together with the target): The RT differences under the corresponding conditions were less than 50 ms. Moore, Yantis, and Vaughan (1998) showed an RT difference of about $50 \mathrm{~ms}$ between the with-occluder and no-occluder conditions for corresponding invalid-same and invalid-different trials. Our results are comparable to theirs, and both results suggest that the processing of amodal completion is not directly reflected in an increase in RT. Just as in the case of feature search, that an increase in set size does not increase search RT is suggestive of preattentive/ parallel processing of features; it is possible that amodal completion, which has been suggested to occur preattentively (Mattingley et al., 1997; Rensink \& Enns, 1998), also occurs in parallel with other preattentive processes. The sudden presence of the occluder and the target may summon the reentrant process of attention (Di Lollo et al., 2000), and while attention is arriving at the target and the occluder, amodal completion is developing in parallel. By the time attention arrives, amodal completion is completed, and the just-arrived attentional reentrant process can then interact with the amodally completed object representation to resample the object configuration and affect motor programming, which has been shown to be modifiable (Goodale, Pelisson, \& Prablanc, 1986; Schettino, Adamovich, \& Poizner, 2003) in response to the presence of a target.

The RT patterns observed in the experiments also hint at this possibility. If one compares the RT in each condition in Experiment 2, where the occluder was presented at the final frame, with that in Experiment 1, where the occluder was presented $100 \mathrm{~ms}$ before target onset, one finds that there is a small increase for each trial type. As compared with Experiment 1, the RTs in Experiment 2 were $20 \mathrm{~ms}$, $F(1,53)=1.453, p=.233,46 \mathrm{~ms}, F(1,53)=7.596$, $p=.008$, and $42 \mathrm{~ms}, F(1,53)=5.14, p=.027$, longer for the valid, invalid-same, and invalid-different trials, respectively. This RT pattern - in addition to reflecting a possible interference from the simultaneously presented occluder with the target (i.e., the task difficulty is increased)suggests that amodal completion occurs in a partially parallel way. Had amodal completion occurred serially, the RT differences should have reflected the $100 \mathrm{~ms}$ needed for amodal completion.

Furthermore, this parallel processing can be operative immediately, before any conscious perception of the 
amodally completed objects. It is commonly accepted that it takes some time for the observer to become aware of the object in view. Wu, Busch, Fabre-Thorpe, and VanRullen (2009) presented a flash probe to bring a physically present but faded-away object back to consciousness in a motioninduced-blindness paradigm. They showed that an alreadyfaded object can return to consciousness earlier than the observer's awareness of the flash probe, and it was calculated that the unconscious object enjoyed a $100-\mathrm{ms}$ temporal advantage over the flash probe. This suggests that from the presentation of a flash probe to the time it is consciously perceived, there is a $100-\mathrm{ms}$ interval of unconscious processing. Referring to our paradigm, when the target and the occluder suddenly appear and before the observer is aware of their presence, there is a $100-\mathrm{ms}$ interval of unconsciousness processing of amodal completion that has already been undertaken. Suppose that detection of a target requires awareness of it; the seeming paradox that amodal completion occurs in no time may not be so contradictory to a $75-$ to $100-\mathrm{ms}$ amodal completion time after all (Murray et al., 2001; Sekuler \& Pratt, 1992).

Finally, our use of a detection task here may have lessened the requirement of a complete object representation for object-based attention to occur. Shomstein and Behrmann (2008) showed that the preview time required for object-based attention to occur is influenced by task type: a longer preview time for a discrimination task and a shorter preview time for a detection task. In other words, the strength of object representation needed for objectbased attention may be different for different tasks. It is possible that the quality of amodal completion required in our detection task is less restricted than that in previous studies using a discrimination task (Chen, Liu, Chen, \& Fang, 2009; Sekuler \& Pratt, 1992). This might be another reason that we were able to observe the influence from amodal completion even when we presented the occluder together with the target.

\section{The dynamic-updating hypothesis}

Our results can be explained by the reentrant process (Di Lollo et al., 2000) that is operative on the changed object configuration. Di Lollo et al. found deterioration of target identification by a common-onset-late-offset mask, which is explained by a reentrant process in which the representation of the target is substituted by that of the mask within a short period of time (e.g., $100 \mathrm{~ms}$ ). Here, we propose that the reentrant process can also explain the interactive process between attention and the representation that is dynamically reorganized even after object recognition has been achieved. In this regard, it is proposed that attention reenters through the feedback loop to monitor the object representation throughout the trial. When a change occurs (such as the amodal completion induced by the occluder in the present study), the reentrant process is boosted in a way similar to that in Experiments 5 and 6 of Dilollo et al., where a precue and a pop-out target were used, respectively. That is, in our manipulation, the iterations needed to update and confirm the changed representation were reduced by the appearance of the change. The key point of the dynamic-updating hypothesis is that attention does not select on a stable object representation that cannot be changed in terms of boundary reorganization (through merge or split). Rather, attention selects on a dynamically evolving representation that can be realized by mechanisms such as the reentrant processing.

Take Experiment 3, for example. Initially, when the cue is presented at one end of the objects, attention is oriented to the cued location. The cued-object configuration would affect how attention would be deployed if there were no further change occurring to the cued object. When the change occurs (i.e., the presence of an occluder), it induces attention to reenter to the cued object configuration. While attention is being reentrant and before it arrives at the cued object configuration, processes such as amodal completion are developing in parallel. When attention arrives, the parallel processes are already completed; thus, attention now selects on a representation that has already integrated changes into it. That is, attention now selects the target appearing at the invalid-same location that, after amodal completion, belongs to the same object as the cued object. In this way, changes occurring on the object after attention is cued to it can exert their influence on attention.

The dynamic-updating hypothesis provides an alternative interpretation of the finding in Moore and Fulton (2005). We demonstrated that the presentation of the occluder at the target frame can influence object-based attention, and this suggests the possibility that the object representation is dynamically reorganized even after attention is cued to the original organization of the scene. In Moore and Fulton's study, two rectangles were each partially overlapped with an occluder that diagonally covered one end of each rectangle in the initial display. After the cue appeared on the visible end of one rectangle, the occluder started to rotate to reveal the occluded ends. After that, the occluder either returned to cover the same ends of the rectangles again ("occluder returned") or left the occluder visible either by placing the occluder in the middle of the two objects ("occluder removed," Experiment 1A) or by moving the occluder back but beneath the two objects, leaving the previously occluded portion visible ("occluder removed," Experiment 1B). The same-object effect was obtained only when the target appeared on the now visible but originally occluded portion (i.e., the "occluder removed" conditions in Experiments 1A and 1B), but not when the target appeared on the same location but on top of 
the returned occluder. They showed that once an object was selected, the selection benefit of attention could extend to the hidden part of the same object. They further suggested that this benefit arises from attentional selection on an organized representation of scenes (i.e., amodal completion by the occluder) in the initial display. That is, attention selects on the object representation of an amodally completed object including the occluded part at the time when the cue just appears. On the basis of the dynamicupdating hypothesis, we suggest that instead of selecting the invisible part of an "intact" object implied by the occlusion at the time when the cue display appears, it is possible that attention initially selects on an incomplete object. Later, the revealed occluded part can be updated into the initially incomplete object and can make the object become a completed one. Then, attention can reenter and process the now fully revealed and intact object.

Having said that, our dynamic-updating hypothesis is in line, in fact, with the object-mediating updating hypothesis of Moore et al. (2007), and we further extend the updating process to relations between objects. Moore et al. (2007) showed that the sudden size change of a moving disc induced an illusory perception of two discs. They suggested that this reveals a breakdown of an object-mediated updating process that is normally working behind the scene. In addition to size changes, we suggest that the visual system also updates the relations between individual objects when objects are merged (as we showed here) or when they are split (Noles, Scholl, \& Mitroff, 2005).

Furthermore, in the context of object updating, the dynamic-updating hypothesis can be viewed as an extension of the cued-object hypothesis. Indeed, without updating the cued object onto which attention was initially deployed, the changed object configuration would be equal to a new representation (after the presence of the occluder), so that no location or object effect should be expected. More important, however-above and beyond the idea of how attention interacts with the object in a conventional view of serial processing, such that a stabilized object representation needs to precede attentional selection - the dynamic-updating hypothesis suggests, instead, a partially parallel process in which attention can operate on an object representation that is constantly being updated. Whether the updating process can be a form of complete overwriting of previously existing representation or whether it requires a certain space-time continuity awaits further study.

Implications for existing theories of object-based attention

Our results in this study provide initial supporting evidence for the attentional focusing hypothesis proposed by Goldsmith and Yeari (2003) and hint at the possible validity of their argument as described below. On the basis of the finding that the same-object effect was found only when attentional focus was wide open, Goldsmith and Yeari suggested that attentional focusing can modulate the quality of the object representation upon which attention selects (thus affecting object-based attention). Although, in all their experiments, they manipulated attentional focusing only in such a way that it was voluntarily maintained wide open or narrowly focused from the beginning of a trial and throughout the trial, they argued that the dynamic change of the spatial extent of attentional focusing after the initial attentional deployment (i.e., cuing) continues to influence object-based attention via modulating the quality of the object representation. In fact, if the object representation after being cued (whether changed or not) were not important for the ongoing attentional process (as suggested by the cued-object hypothesis), there would be no role for the dynamic attentional focusing change to play in objectbased attention after the initial attentional deployment. As we showed, the postcuing object representation is also important; this overcomes the aforementioned concern. In addition, since we presented the occluder as an abrupt onset in our three experiments, it might be that the attentional focusing is changed in response to the presence of the occluder accordingly. However, to confirm this and thus confirm the argument of Goldsmith and Yeari that attentional focusing affects object-based attention not only from the beginning of a trial, but also beyond the time of attentional deployment and throughout the trial, further studies can measure whether attentional focusing indeed is changed either involuntarily, as in our case, or voluntarily in other postcuing changes.

One implication of our dynamic-updating hypothesis is that the interaction between attention and an object is dynamic; thus, when hypothesizing how attention selects an object for further processing, the accounts that presume a stable relation between attention and an object require modifications in order to adequately explain our results here. That is, existing accounts need to incorporate the temporal aspects of how attention interacts with an object if the object is changed after attention is oriented to the cued object.

There are three main accounts for the object-based attention: the spreading account (Egly et al., 1994), the prioritization account (Shomstein \& Yantis, 2002), and the shift account (Lamy \& Egeth, 2002). They differ with regard to the point of time at which object-based attention takes effect. The attentional-spreading hypothesis (Egly et al., 1994; Richard, Lee, \& Vecera, 2008) states that attentional resources spread more efficiently within a cued object than between objects. Thus, the spreading of attention can start as early as the time at which the cue lands on the object. The attentional shift account (Lamy \& Egeth, 2002) states that it is more difficult for attention to 
shift between objects than within an object when the targets appear. Unlike the attentional-spreading hypothesis, which assumes that attention spreads immediately after presentation of the cue display, such a mechanism of shift cost probably comes into play at the time when the target appears. Finally, the prioritization account (Shomstein \& Yantis, 2002) suggests that uncertainty about target location induced a serial scanning of objects, ${ }^{1}$ which results in the attended object being processed with higher priority than the not-yet-attended objects. It can be viewed as an overall strategic setting based on a buildup of knowledge of the relation between the cue and target (i.e., cue validity); thus, according to the prioritization account, attention can also start to operate as early as when the cue appears.

Our results would affect the spreading account most because the change we introduced (Experiments 2 and 3) occurred after the time point that attention, presumably, starts to spread. Furthermore, in our design (e.g., in Fig. 1, where the amodally completed objects are vertically oriented), attention spreads across more of the area that is occupied by the objects along one direction than along the other direction; thus, the spreading account predicts the same-object effect even without the presence of the occluder. However, in Experiment 3, we obtained the same-object effect only in the occluder-above condition, but not in the occluder-below condition. Altogether, to explain our results, the spreading account needs to be modified in such a way that the efficiency of spreading is different between the occluder-above and occluder-below conditions and this difference in efficiency can be operative at the final target frame when the occluder is presented. For the shift account, because attention shifts when the target appears, it would be possible to explain our data if the configuration of objects is recalculated at the final frame before attention starts to shift- that is, if one's attention can "look before it jumps." For the prioritization account to explain our results, the priority assigned to the originally cued object should be able to be shared or extended to the amodally completed object, because the objects are merged after the cue display in our study. In sum, only by incorporating into these accounts the fact that changes about objects affect attention can we extend our understanding of object-based attention via these accounts.

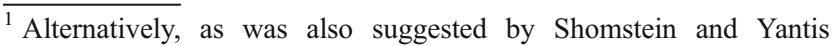
(2002), attentional prioritization can be achieved by "display-wide parallel processing with different rates of information extraction according to attentional priority" (Shomstein \& Yantis, 2002, footnote). Either serial or parallel, the attentional prioritization account faces the same situation as that demonstrated in the present study, that the priorities assigned to the objects are changed when the objects are merged via amodal completion.
}

\section{Conclusion}

The present study focused on the dynamic aspect of the object that is selected by attention and showed that attention does not select a stable representation that cannot be changed once attention has been deployed to it. Future studies can be done to examine the updating process underlying the dynamic-updating hypothesis to further our understand of how attention selects an object and interacts with it in a constantly changing visual world. After all, in the face of the constant changes in the visual world, above and beyond the first comer that attracts attention, it happens more often that the one who stays last gets attended.

Acknowledgments This research was supported by grants from Taiwan's National Science Council (NSC96-2752-H-002-008-PAE, NSC 96-2413-H-002-009-MY3, and NSC 98-2410-H-002-023-MY3). We thank James Brown, Anne Hillstrom, Jay Pratt, Yaffa Yeshurun, and an anonymous reviewer for their helpful comments and suggestions on earlier versions of the manuscript.

\section{References}

Abrams, R. A., \& Law, M. B. (2002). Random visual noise impairs object-based attention. Experimental Brain Research, 142, 349-353.

Alvarez, G. A., \& Scholl, B. J. (2005). How does attention select and track spatially extended objects? New effects of attentional concentration and amplification. Journal of Experimental Psychology: General, 134, 461-476.

Behrmann, M., Zemel, R. S., \& Mozer, M. C. (1998). Object-based attention and occlusion: Evidence from normal participants and a computational model. Journal of Experimental Psychology: Human Perception and Performance, 24, 1011-1036.

Benso, F., Turatto, M., Mascetti, G. G., \& Umiltà, C. (1998). The time course of attentional focusing. European Journal of Cognitive Psychology, 10, 373-388.

Chen, J., Liu, B., Chen, B., \& Fang, F. (2009). Time course of amodal completion in face perception. Vision Research, 49, 752-758.

Di Lollo, V., Enns, J. T., \& Rensink, R. A. (2000). Competition for consciousness among visual events: The psychophysics of reentrant visual processes. Journal of Experimental Psychology: General, 129, 481-507.

Duncan, J. (1984). Selective attention and the organization of visual information. Journal of Experimental Psychology: General, 113, 501-517.

Egly, R., Driver, J., \& Rafal, R. D. (1994). Shifting visual attention between objects and locations: Evidence from normal and parietal lesion subjects. Journal of Experimental Psychology: General, 123, 161-177.

Eriksen, C. W., \& Yeh, Y. Y. (1985). Allocation of attention in the visual field. Journal of Experimental Psychology: Human Perception and Performance, 11, 583-597.

Forster, K. I., \& Forster, J. C. (2003). DMDX: A Windows display program with millisecond accuracy. Behavior Research Methods, Instruments, \& Computers, 35, 116-124.

Goldsmith, M., \& Yeari, M. (2003). Modulation of object-based attention by spatial focus under endogenous and exogenous orienting. Journal of Experimental Psychology: Human Perception and Performance, 29, 897-918. 
Goodale, M. A., Pelisson, D., \& Prablanc, C. (1986). Large adjustments in visually guided reaching do not depend on vision of the hand or perception of target displacement. Nature, 320, 748-750.

Ho, M. C., \& Yeh, S. L. (2009). Effects of instantaneous object input and past experience on object-based attention. Acta Psychologica, 132, 31-39.

Kahneman, D., Treisman, A., \& Gibbs, B. J. (1992). The reviewing of object files: Object-specific integration of information. Cognitive Psychology, 24, 175-219.

Kellie, F. J., \& Shapiro, K. L. (2004). Object file continuity predicts attentional blink magnitude. Perception \& Psychophysics, 66, 692712 .

Kravitz, D. J., \& Behrmann, M. (2008). The space of an object: Object attention alerts the spatial gradient in the surround. Journal of Experimental Psychology: Human Perception and Performance, 34, 298-309.

Lamy, D., \& Egeth, H. (2002). Object-based selection: The role of attentional shifts. Perception \& Psychophysics, 64, 52-66.

Lamy, D., \& Tsal, Y. (2000). Object features, object locations, and object files: Which does selective attention activate and when? Journal of Experimental Psychology: Human Perception and Performance, 26, 1387-1400.

Macquistan, A. (1997). Object-based allocation of visual attention in response to exogenous, but not endogenous, spatial precues. Psychonomic Bulletin \& Review, 4, 512-515.

Mattingley, J. B., Davis, G., \& Driver, J. (1997). Preattentive filling-in of visual surfaces in parietal extinction. Nature, 275, 671-674.

Moore, C. M., \& Fulton, C. (2005). The spread of attention to hidden portions of occluded surfaces. Psychonomic Bulletin \& Review, 12, 301-306

Moore, C. M., Mordokoff, J. T., \& Enns, J. T. (2007). The path of least persistence: Object status mediates visual updating. Vision Research, 47, 1624-1630.

Moore, C. M., Yantis, S., \& Vaughan, B. (1998). Object-based visual selection: Evidence from perceptual completion. Psychological Science, 9, 104-110.

Murray, R. F., Sekuler, A. B., \& Bennett, P. J. (2001). Time course of amodal completion revealed by a shape discrimination task. Psychonomic Bulletin \& Review, 8, 713-720.
Noles, N. S., Scholl, B. J., \& Mitroff, S. R. (2005). The persistence of object file representations. Perception \& Psychophysics, 67, 324-334.

Posner, M. I., Snyder, C. R., \& Davidson, B. J. (1980). Attention and the detection of signals. Journal of Experimental Physiology, $109,160-174$

Pratt, J., \& Sekuler, A. B. (2001). The effects of occlusion and past experience on the allocation of object-based attention. Psychonomic Bulletin \& Review, 8, 721-727.

Pylyshyn, Z. W., \& Storm, R. W. (1988). Tracking multiple independent targets: Evidence for a parallel tracking mechanism. Spatial Vision, 3, 179-197.

Raymond, J. E. (2003). New objects, not new features, trigger the attentional blink. Psychological Science, 14, 54-59.

Rensink, R. A., \& Enns, J. T. (1998). Early completion of occluded objects. Vision Research, 38, 2489-2505.

Richard, A. M., Lee, H., \& Vecera, S. P. (2008). Attentional spreading in object-based attention. Journal of Experimental Psychology: Human Perception and Performance, 34, 842-852.

Schettino, L. F., Adamovich, S. V., \& Poizner, H. (2003). Effects of object shape and visual feedback on hand configuration during grasping. Experimental Brain Research, 151, 158-166.

Scholl, B. J. (2001). Objects and attention: The state of the art. Cognition, 80, 1-46.

Sekuler, A. B., \& Palmer, S. E. (1992). Perception of partly occluded objects: A microgenetic analysis. Journal of Experimental Psychology: General, 121, 95-111.

Shomstein, S., \& Behrmann, M. (2008). Object-based attention: Strength of object representation and attentional guidance. Perception \& Psychophysics, 70, 132-144.

Shomstein, S., \& Yantis, S. (2002). Object-based attention: Sensory modulation or priority setting? Perception \& Psychophysics, 64, $41-51$.

Shomstein, S., \& Yantis, S. (2004). Configural and contextual prioritization in object-based attention. Psychonomic Bulletin \& Review, 11, 247-253.

Vecera, S. P., \& Farah, M. J. (1994). Does visual attention select locations or objects? Journal of Experimental Psychology: General, 123, 146-160.

Wu, C. T., Busch, N. A., Fabre-Thorpe, M., \& VanRullen, R. (2009). The temporal interplay between conscious and unconscious perceptual streams. Current Biology, 19, 2003-2007. 